\title{
THE ESSENTIAL SET OF FUNCTION ALGEBRAS
}

\author{
ROBERT E. MULLINS ${ }^{1}$
}

Let $X$ be a compact Hausdorff space and $C(X)$ the Banach algebra of all complex-valued continuous functions on $X$ under the sup-norm. By a function algebra $A$ we mean a closed subalgebra of $C(X)$ which separates points and contains the constant functions. Let $S(A)$ denote the space of maximal ideals of $A$ and let $E(A)$ denote the essential set of $A$ as defined by Bear [1], i.e. $E(A)$ is the hull of the largest closed ideal of $C(X)$ which is contained in $A$. We will obtain another characterization of the essential set in the case when $S(A)=X$ and thereby obtain some results of a global nature from local hypotheses.

TheOREM 1. Let $A$ be a function algebra on a compact metric space $X$. Suppose $X=S(A)$ and let $E$ be the essential set of $A$ in $X$. If $x_{0}$ has a neighborhood $V$ such that $A \mid \bar{V}=C(\bar{V})$ then $x_{0} \notin E$.

Proof. Let $V$ be a neighborhood of $x$ such that $A \mid \bar{V}=C(\bar{V})$. Then $x_{0}$ is a local peak point, and hence [2, Theorem 4.1] $x_{0}$ is a global peak point. Let $f \in A, f\left(x_{0}\right)=1$ and $|f(x)|<1$ if $x \neq x_{0}$. Let $r$ be a number so close to one that

$$
U=\{x: \operatorname{Re} f(x) \geqq r\} \subset V .
$$

Then $\operatorname{Re} f(x) \leqq s<r<1$ for all $x \in X \sim V$. Let $p_{n}$ be a sequence of polynomials which converge uniformly on $f[U] \cup f[X \sim V]$ to a function one on $f[U]$ and zero on $f[X \sim V]$ (see e.g. Wermer: Banach algebras and analytic functions, Theorem 7.6). Then $p_{n} \circ f$ converges uniformly on $X$, except possibly on $V \sim U$, to a function one at $x_{0}$ and zero off $V$. Let $g \in A, g\left(x_{0}\right)=1$ and $g \equiv 0$ on $V \sim U$. Then $g\left(p_{n} \circ f\right)$ converges uniformly on $X$ to a function $k \in A$ such that $k\left(x_{0}\right)=1$, and $k \equiv 0$ off $U$. Let $W$ be a neighborhood of $x$ such that $k \neq 0$ on $W$. Let $h$ be any continuous function on $X$ which is zero off $W$. Let $w \in A$ with $w=h / k$ on $W$. Then $h=w k \in A$. That is, $A$ contains every continuous function zero off $W$. Hence $X \sim W \supset E$, and $x_{0} \notin E$.

Corollary. Let $A$ be a function algebra on the compact metric space $X=S(A)$ and let $E$ be the essential set of $A$ in $X$. Then $E=X \sim P$, where $P=\{x \in X: A \mid \bar{V}=C(\bar{V})$ for some neighborhood $V$ of $x\}$.

Received by the editors January 27, 1966.

1 Part of the material in this paper appears in the author's doctoral dissertation at Northwestern University. This research was supported in part by the National Science Foundation. 
ThEOREM 2. Let $A$ be a function algebra on a compact metric space $X=S(A)$. Let $F_{1}, F_{2}, \cdots$ be a sequence of closed sets such that $X=\bigcup_{i=1}^{\infty} F_{i}$ and $A \mid F_{i}$ is closed in $C\left(F_{i}\right)$ for $i=1,2, \cdots$. Then $\bar{\cup}_{i=1}^{\infty} E_{i}$ is the essential set of $A$ in $X$ where $E_{i}$ is the essential set of $A \mid F_{i}$ in $F_{i}$.

Proof. Let $E$ denote the essential set of $A$ in $X$ and let $I$ denote the largest closed ideal of $C(X)$ contained in $A$. Let $J_{i}$ be the largest ideal of $C\left(F_{i}\right)$ which is contained in $A \mid F_{i}$. Clearly $I \mid F_{i}$ is contained in $J_{i}$. Thus for $i=1,2, \cdots, E_{i}=\left\{x \in F_{i}: f(x)=0\right.$ for all $f$ in $\left.J_{i}\right\}$ is contained in $E=\{x \in X: f(x)=0$ for all $f$ in $I\}$. Since $E$ is closed, $\bar{U}_{i=1}^{\infty} E_{i} \subset E$. Let $U=E \sim \bar{U} E_{i}$. It is shown in Bear [1] that $A \mid E$ is a function algebra with essential set $E$ and maximal ideal space $E$. Since $U=\bigcup_{i=1}^{\infty}\left(U \cap F_{i}\right)$ is open in $E$, it follows from the Baire Category theorem that some $U \cap F_{j}$ has a nonempty interior in $E$. Let $V$ be a closed neighborhood, relative to $E$, which is contained in $U \cap F_{j}$. Since $V$ is disjoint from $E_{j}$, the essential set of $A \mid F_{j}$, it follows that $A \mid V=C(V)$. This contradicts the result of Theorem 1, namely that $P=\left\{x \in E: A \mid V_{x}=C\left(V_{x}\right)\right.$ for some closed neighborhood $V_{x}$ of $\left.x\right\}$ must be the empty set.

Corollary. Let $A$ be a function algebra on the compact metric space $X=S(A)$. Let $F_{1}, F_{2}, \cdots$ be a sequence of closed sets such that $X$ $=\bigcup_{i=1}^{\infty} F_{i}$ and $A \mid F_{i}=C\left(F_{i}\right)$ for $i=1,2, \cdots$. Then $A=C(X)$.

If $X$ is a union of a finite number of $F_{i}$ and if $A \mid F_{i}=C\left(F_{i}\right)$, it is a necessary consequence that $S(A)=X$ as the following lemma shows.

Lemma. Let $X$ be a compact Hausdorff space and let $F_{1}, \cdots, F_{n}$ be $n$ closed sets such that $X=\bigcup_{i=1}^{n} F_{i}$. If $A$ is a function algebra on $X$ such that $A \mid F_{i}$ is closed in $C\left(F_{i}\right)$ and $S\left(A \mid F_{i}\right)=F_{i}$ for $i=1,2, \cdots, n$ then $S(A)=X$.

Proof. Let $M$ be a proper maximal ideal in $A$. If $M\left|F_{i}=A\right| F_{i}$ for $i=1,2, \cdots, n$, there exist functions $f_{1}, \cdots, f_{n}$ in $M$ such that $f_{i}(x)=1$ whenever $x$ is in $F_{i}$. Let $h_{2}=f_{1}+f_{2}-f_{1} f_{2}$. Assuming $h_{j}$ has been defined let $h_{j+1}=f_{j+1}+h_{j}-f_{j+1} h_{j}$. We thus get a function $h_{n}$ in $M$ such that $h_{n}$ is one on $X$. This contradicts the assertion that $M$ is a proper ideal. There must therefore exist an integer $k(k \leqq n)$ such that $M \mid F_{k}$ is a proper ideal in $A \mid F_{k}$. The ideal $M \mid F_{k}$ is contained in a maximal ideal of $A \mid F_{k}$. Thus there exists $x_{0}$ in $F_{k}$ such that $M \mid F_{k}$ $\subset\left\{f \in A \mid F_{k}: f\left(x_{0}\right)=0\right\}$. It then follows that $M$ corresponds to evaluation at $x_{0}$. This completes the proof that $S(A)=X$.

Theorem 3. Let $A$ be a function algebra on a compact metric space $X$. 
Let $F_{1}, \cdots, F_{n}$ be $n$ closed sets such that $X=\bigcup_{i=1}^{n} F_{i}$ and $A \mid F_{i}=C\left(F_{i}\right)$ for $i=1, \cdots, n$. Then $A=C(X)$.

Proof. Since $S(A)=X$ by the above lemma, this theorem is precisely the corollary after Theorem 2 in the finite case.

Corollary. Let $A$ be a function algebra on a compact metric space $X$. If for each $x$ in $X$ there exists a closed neighborhood $V_{x}$ of $x$ such that $A \mid V_{x}=C\left(V_{x}\right)$, then $A=C(X)$.

I wish to thank the referee for supplying a proof of Theorem 1, much shorter than the proof I originally submitted.

\section{REFERENCES}

1. H. S. Bear, Complex function algebras, Trans. Amer. Math. Soc. 90 (1959), 383-393.

2. H. Rossi, The local maximum modulus theorem, Ann. of Math. 72 (1960), 1-11.

NORTHWESTERN UNIVERSITY AND

MARQUETTE UNIVERSITY 